Research Article

\title{
Antibiotic sensitivity pattern of Staphylococcus aureus in a tertiary care hospital of Sri Lanka.
}

\author{
Jayatilleke $\mathrm{K}^{1}$, Bandara $\mathrm{P}^{1}$ \\ Sri Lanka Journal of Infectious Diseases 2012 Vol.2(2);13-17 \\ DOI: http://dx.doi.org/10.4038/sljid.v2i2.4162
}

Key words: Staphylococcus aureus, MRSA, ABST

\begin{abstract}
The aim of this study was to determine the antibiotic sensitivity pattern of Staphylococcus aureus in a tertiary care hospital of Sri Lanka. $S$ aureus isolated from patient samples received during the period April to December 2010 were identified by standard methods. The antimicrobial susceptibility testing was performed using the standard disc diffusion method recommended by the Clinical and Laboratory Standards Institute 2008. Of $431 \mathrm{~S}$ aureus isolates from all specimens received during this period, 203(47\%) were methicillin resistant (MRSA), 45 of which were from screening swabs. Of the 210 methicillin sensitive $S$ aureus (MSSA) isolates tested for penicillin sensitivity, 83\% were resistant. Erythromycin and clindamycin sensitivity were tested in 149 isolates with $30.9 \%$ and $17.5 \%$ resistance respectively. Resistance to ciprofloxacin was $12 \%$ in 108 isolates tested. Erythromycin, clindamycin and ciprofloxacin were not tested for all the isolates due to non-availability of antibiotic discs.

Erythromycin and clindamycin resistance in 164 MRSA isolates was $79 \%$ and $72 \%$ respectively. Ciprofloxacin resistance in 125 MRSA isolates tested was 54\%. Gentamicin resistance of 44\% ( $n=127$ isolates) and cotrimoxazole resistance of $48 \%(n=158$ isolates) was noted. All MRSA isolates were sensitive to vancomycin, teicoplanin and linezolid.

There were 18 isolates of $S$ aureus from blood cultures of 16 patients. Only 2 of 17 isolates tested were sensitive to penicillin. 7 of $18(38.9 \%)$ isolates were MRSA. 50\% of these isolates were resistant to erythromycin while $25 \%$ were resistant to clindamycin. $23.5 \%$ were resistant to ciprofloxacin.
\end{abstract}

${ }^{1}$ Sri Jayewardenapura General hospital, Nugegoda, Sri Lanka

Address for correspondence: Dr Kushlani Jayatilleke, 393/c, 3rd Lane, Dutugemunu Mawatha, Battaramulla, Sri Lanka.Tel: 00094714145056 Email: kush jaya@yahoo.co.uk 
Of the 45 strains isolated from screening swabs, 18\% were MRSA. As a high proportion of $S$ aureus is MRSA in this hospital setting, it is important to do culture and sensitivity of relevant specimens when $S$ aureus infection is suspected. The results of this study suggest that MRSA cover may need to be considered in empirical therapy of possible $S$ aureus infections.

\section{Introduction}

Annually, nosocomial infections account for morbidity and mortality among millions of patients, worldwide. ${ }^{1}$ Methicillin-resistant Staphylococcus aureus (MRSA) is relatively ubiquitous and the cause of community acquired colonization and infections as well as endemic and epidemic nosocomial colonization and infections. ${ }^{1}$

MRSA is of concern not only because of its resistance to methicillin, but also because of its general resistance to many other chemotherapeutic agents. Staphylococcal cassette chromosome mec ( $\mathrm{SCCmec}$ ) is a genomic island of unknown origin containing the antibiotic resistance gene $m e c \mathrm{~A} .^{2}$ mecA is responsible for resistance to methicillin and other $\beta$-lactam antibiotics. mecA encodes penicillin-binding protein $2 \mathrm{a}$ (PBP2a), which differs from other penicillin-binding proteins as its active site does not bind methicillin or other $\beta$-lactam antibiotics. As such, PBP2a can continue to catalyze the transpeptidation reaction required for peptidoglycan cross-linking, enabling cell wall synthesis in the presence of antibiotics that act on cell wall synthesis. ${ }^{2}$ As a consequence of the inability of PBP2a to interact with $\beta$-lactam moieties, acquisition of mecA confers resistance to all $\beta$-lactam antibiotics including methicillin. Since MRSA strains are also resistant to multiple antibiotics there is a possibility of causing extensive outbreaks, which may be difficult to control. ${ }^{3}$ Accurate detection of MRSA is therefore an important prerequisite for appropriate therapy and epidemiological assessment of nosocomial infections caused by this organism. ${ }^{3,4}$

After the emergence of MRSA as a nosocomial pathogen in the early 1960 s $^{5}$ an increasing number of outbreaks due to MRSA infections in hospitals have been reported from many countries, ranging from abscesses to life-threatening sepsis, endocarditis, and osteomyelitis. ${ }^{6}$

Currently, therapeutic options for MRSA infections are limited to a very few expensive and potentially toxic drugs like teicoplanin, vancomycin , linezolid, daptomycin and streptogramins. Thus, control of MRSA is essential to curtail the introduction and spread of infection. ${ }^{7}$ Early detection of MRSA and formulation of an effective antibiotic policy, along with infection control in tertiary care hospitals is of paramount importance from an epidemiological viewpoint.

This study has been carried out in our hospital with the aim of determining the antibiotic susceptibility of $S$ aureus, in order to utilize that information to formulate antibiotic policy and appropriate control measures.

\section{Methods}

This study was carried out in a tertiary care hospital in Sri Lanka from $1^{\text {st }}$ April 2010 to $31^{\text {st }}$ December 2010. All S. aureus isolates $(\mathrm{n}=431)$ obtained during this 9 month period were included in the study. These were isolated from various clinical samples including pus, sputum, 
urine, high vaginal swabs, blood, and body fluids and also from screening swabs for MRSA from hospital inpatients. Screening swabs were inoculated into a $7 \%$ sodium chloride solution on day one and subcultured after overnight incubation at $35^{\circ} \mathrm{C}$ onto Blood agar and MacConkey agar. All other samples were directly inoculated onto blood agar and MacConkey agar plates and incubated aerobically at $35^{\circ} \mathrm{C}$ for 24 hours. The isolates were identified with standard tests used to identify $S$ aureus such as Gram stain, catalase, slide and tube coagulase and Staphylase (Oxoid) tests. Antibiotic sensitivity testing was performed using the standard disc diffusion method recommended by the Clinical and Laboratory Standard Institute $(2008)^{8}$ for the following antibiotics:, ciprofloxacin, chloramphenicol, clindamycin, gentamicin, erythromycin, penicillin , rifampicin, vancomycin, teicoplanin and linezolid. Inducible clindamycin resistance was tested by D-zone as recommended in the CLSI guidelines (2008). ${ }^{8}$ Testing for methicillin resistance was performed using the standard disc diffusion method recommended by the Clinical and Laboratory Standard Institute $(2008)^{8}$ with cefoxitin $30 \mu \mathrm{g}$ disc on Mueller-Hinton agar with 24 hours incubation at $35^{\circ} \mathrm{C}$. Results were interpreted according to the criteria of CLSI 2008. ${ }^{8}$ Multiple colonies were used to prepare the inoculum. $S$ aureus ATCC 25923 was used as the quality control strain .

\section{Results}

There were 431 isolates of $S$ aureus, of which 386 were from clinical samples and 45 from screening swabs. MRSA constituted $47 \%$ of the total. MRSA constituted $50.5 \%(\mathrm{n}=195)$ of $S$ aureus isolated from clinical samples, and $17.8 \%(\mathrm{n}=8)$ from screening swabs.

Table 1 : Resistance of $S$ aureus to commonly used antibiotics*

\begin{tabular}{|l|c|c|c|c|}
\hline & \multicolumn{2}{|c|}{ MSSA $\mathrm{n}=228$} & \multicolumn{2}{c|}{ MRSA $\mathrm{n}=203$} \\
\hline & No tested & $\%$ resistant & No tested & $\begin{array}{c}\% \\
\text { resistance }\end{array}$ \\
\hline Penicillin & 210 & 83 & 167 & 100 \\
\hline Erythromycin & 149 & 30.9 & 164 & 79 \\
\hline Clindamycin & 149 & 17.5 & 164 & 72 \\
\hline Ciprofloxacin & 108 & 12 & 125 & 54 \\
\hline Gentamicin & 102 & 2 & 127 & 44 \\
\hline Cotrimoxazole & 118 & 6 & 158 & 48 \\
\hline
\end{tabular}

*Number tested varied according to availability of antibiotic discs

All the isolates tested were sensitive to vancomycin and teicoplanin.

There were 18 isolates of $S$ aureus from blood cultures of 16 patients. Only 2 of 17 isolates tested were sensitive to penicillin. 7 of the 18 (38.9\%) isolates were MRSA. 50\% of $S$ aureus from blood cultures were resistant to erythromycin while $25 \%$ were resistant to clindamycin. $23.5 \%$ were resistant to ciprofloxacin. All the isolates were sensitive to vancomycin, teicoplanin and linezolid. 


\section{Discussion}

Despite intensive efforts to control resistant organisms by aggressive infection control methods, antibiotic-resistant staphylococci, especially MRSA have become the most common cause of hospital acquired infections worldwide. ${ }^{1}$

The present study highlights the problem of MRSA in this hospital as well as in Sri Lanka. As this is a tertiary care hospital and there is no proper referral system in the country, patients treated in different types of hospitals from different parts of the country are transferred to the study hospital for specialized treatment. In this study, the prevalence of MRSA among $S$ aureus isolates was $47 \%$. This is high in comparison to records of other hospitals in the world. In India, the MRSA isolation rate ranges from $29 \%$ to $39.5 \% .^{9,10}$ Iran has a similar range with $35 \%$ of MRSA. ${ }^{11}$ In a study carried out in the National Hospital of Sri Lanka $86 \%$ of the S. aureus isolates from wound curettings were MRSA. ${ }^{12}$

The annual report of the European antimicrobial resistance surveillance network in 2009 showed that MRSA blood stream infection in France was 23\% (54 labs and 4727 isolates), Germany 18\% (17 labs and 1894 isolates), the Netherlands < 1\% (16 labs and 1035 isolates) and the United Kingdom 28\% (69 labs and 2977 isolates). In the current study, of the 18 Staphylococcus aureus isolates from blood cultures, $38.9 \%$ were MRSA.

MRSA strains isolated in the current study have shown a high level of resistance to antibiotics such as erythromycin and clindamycin (54\% and $44 \%$ respectively) which are used in the treatment of staphylococcal infections. This is higher than levels of resistance described in earlier studies in India ${ }^{9,10}$ and Iran. ${ }^{11}$ Ciprofloxacin resistance of $34.3 \%$ is however similar to results obtained in $\operatorname{Iran}(33.7 \%){ }^{11}$

Since complete eradication of MRSA may not be possible, control of transmission seems to be an appropriate goal. Prevention of transmission of MRSA is primarily through effective hand hygiene ${ }^{13}$ of health care staff associated with patient management. Regular surveillance with communication of results as obtained in the current study to clinical care givers is essential for the implementation and sustaining of recommended infection control practices. In addition, the use of broad-spectrum antibiotics for treating infections also increases colonization with MRSA and other resistant bacteria. ${ }^{1}$ More effective antibiotic control may also be required to control MRSA.

\section{Conclusion}

As a high proportion of Staphylococcus aureus is MRSA, It is important to do culture and sensitivity of relevant specimens when $S$ aureus infection is suspected. Also MRSA cover needs to be considered in empiric therapy of possible Staphylococcus aureus infections.

\section{References}

1. Mansouri S, Khaleghi M. Antibacterial resistance pattern and frequency of methicillin resistant Staphylococcus aureus . Iranian J Med Sci 1997;22:93. No doi 
2. Lowy D.F. Antimicrobial resistance: the example of Staphylococcus aureus. J Clin Invest. 2003;111(9):1265-1273 doi:10.1172/JCI200318535

3. Majumder D, Sarma Bordoloi JN, Phukan AC, Mahanta J. Antimicrobial susceptibility pattern among methicillin resistant staphylococcus isolates in Assam. Indian $\mathrm{J}$ Med Microbiol 2001;19:138-40. No doi

4. Anupurba S, Sen MR, Nath G, Sharma BM, Gulati AK, Mohapatra TM. Prevalence of methicillin resistant Staphylococcus aureus in a tertiary referral hospital in Eastern Utter Pradesh. Indian J Med Microbiol 2003;21:49-51. No doi

5. Gould I. MRSA in Practice. The Royal Society of Medicine Press, 2006; pp 687-8 ISBN 978-1-85315

6. Cox RA, Conquest C, Mallaghan C, Marples RR. A major outbreak of methicillin resistant Staphylococcus aureus caused by a new phage type (EMRSA-16). J Hosp Infect 1995; 29:87-106 doi:10.1016/0195-6701(95)90191-4

7. Siddiqui F, Madahiah-bint-e-Masood, Noor-us-Saba, Samad A, Quayyum M, Qazilbash AA. Antibiogram sensitivity pattern of Methicillin resistant Staphylococcus aureus isolates from pus sample. Pak J Biol Sci 2002;5:491-3 doi:10.3923/pjbs.2002.491.493

8. Performance standards for Antimicrobial Susceptibility Testing; January 2008, M100-S18, volume 28, No.1.

9. Mulla S, Patel M, Shah L, Vaghela G. Study of antibiotic sensitivity pattern of methicillinresistant Staphylococcus aureus. Indian Journal of Critical Care 2007;11:99-101 doi:10.4103/0972-5229.33394

10. Vidya P, RaoV, Rao SP .Prevalence and antimicrobial susceptibility pattern of methicillinresistant Staphylococcus aureus [MRSA] isolates at a tertiary care hospital in Mangalore, South India. Journal of Laboratory Physicians 2010;2:82-84 doi:10.4103/0974-2727.72155

11. Aghazadeh M, Rahbar M, Monnavar MK, Moghadam FS .Sensitivity pattern of methicillin resistant and methicillin sensitive Staphylococcus aureus isolates, against several antibiotics including tigecycline in Iran: A hospital based study. Pak J Med Sci 2009;25: 443-446. No doi

12. Bandara P.L.L, Corea E.M., Bacteriology of diabetic foot ulcers at the general surgical units of the National Hospital of Sri Lanka; poster presentation, $123^{\text {rd }}$ Annual Scientific Sessions Sri Lanka Medical Association, 2010. No doi

13. Siegel JD, Rhinehart E, Jackson M, Chiarello L, and the Healthcare Infection Control Practices Advisory Committee, 2007 Guideline for Isolation Precautions: Preventing Transmission of Infectious Agents in Healthcare Settings. pp 49 http://www.cdc.gov/ncidod/dhqp/pdf/isolation2007.pdf 